\title{
Ajuste de Modelos Não-Lineares em Estudos de Associação entre Polimorfismos Genéticos e Crescimento em Bovinos de Corte ${ }^{1}$
}

\section{Claudia Cristina Paro de Paz ${ }^{2}$, Irineu Umberto Packer ${ }^{3}$, Alfredo Ribeiro de Freitas ${ }^{4}$, Daniela Tambasco-Talhari ${ }^{5}$, Luciana Correa de Almeida Regitano ${ }^{4}$, Maurício Mello de Alencar ${ }^{4}$, Geraldo Maria da $\mathrm{Cruz}^{4}$}

\begin{abstract}
RESUMO - Foram utilizados dados de peso ao nascimento, ao desmame e mensais dos 8 aos 19 meses de idade de 11 classes de genótipos, formadas pela concatenação dos polimorfismos genéticos da kappa-caseína-HinfI (CSN3): AA e AB, do hormônio do crescimento-AluI (GH): LL e LV e da $\beta$-lactoglobulina-HaeIII (LGB): AA, AB e BB $\left(\mathrm{G}_{1}=\mathrm{AALLAA}, \mathrm{G}_{2}=\mathrm{AALLAB}, \mathrm{G}_{3}=\mathrm{AALLBB}\right.$, $\mathrm{G}_{4}=\mathrm{AALVAB}, \mathrm{G}_{5}=\mathrm{AALVBB}, \mathrm{G}_{6}=\mathrm{ABLLAA}, \mathrm{G}_{7}=\mathrm{ABLLAB}, \mathrm{G}_{8}=\mathrm{ABLLBB}, \mathrm{G}_{9}=\mathrm{ABLVAA}, \mathrm{G}_{10}=\mathrm{ABLVAB}$ e $\left.\mathrm{G}_{11}=\mathrm{ABLVBB}\right)$. As informações foram obtidas de animais de três grupos genéticos: $1 / 2$ Canchim-Nelore (CN), $1 / 2$ Angus-Nelore (AN) e $1 \frac{1}{2} \operatorname{Simental-Nelore~(SN),~}$ nascidos em 1998 e 1999 e pertencentes à Embrapa Pecuária Sudeste, São Carlos, SP. Dos cinco modelos estudados: Brody, Von Bertalanffy, Richards, Gompertz e Logístico, o último apresentou melhor qualidade de ajuste. As estimativas dos parâmetros $A$ (valor assintótico), $k$ (taxa de maturação) e $m$ (ponto de inflexão) obtidas do modelo Logístico, ajustado para descrever o crescimento de cada animal, foram analisadas pelo método dos quadrados mínimos, por meio de um modelo linear, que incluiu, além da média geral, o efeito do genótipo, o ano de nascimento, o sexo e o manejo alimentar. Para os animais do grupo genético CN, os genótipos influenciaram significativamente as estimativas dos parâmetros $A$ e $k$ da curva de crescimento. O genótipo $\mathrm{G}_{3}$ apresentou valor inferior de $A$ e superior de $k$ em relação aos genótipos $\mathrm{G}_{7}$ e $\mathrm{G}_{8}$. Quanto aos grupos genéticos $\mathrm{AN}$ e $\mathrm{SN}$, não foi observado efeito significativo do genótipo sobre nenhum dos três parâmetros. A aplicação da técnica de modelos não-lineares em estudos de associação entre polimorfismos genéticos e crescimento animal proporcionou uma análise detalhada do desenvolvimento dos animais de diferentes genótipos (genes: CSN3, GH e LGB).
\end{abstract}

Palavras-chave: Bos taurus x Bos indicus, curvas de crescimento, desenvolvimento ponderal, marcadores genéticos, modelo Logístico

\section{Adjustment of Nonlinear Models in Study of Association between Genetic Polymorphisms and Growth in Beef Cattle}

\begin{abstract}
Weights at birth, at weaning and monthly from 8 to 19 months of age of the 11 genotype identified by combination of polymorphic RFLP's of the genes kappa-casein-HinfI (CSN3): AA and AB, growth hormone-AluI (GH): LL and LV, and $\beta$-lactoglobulin-HaeIII (LGB): AA, AB and BB ( $\mathrm{G}_{1}=\mathrm{AALLAA}, \mathrm{G}_{2}=\mathrm{AALLAB}, \mathrm{G}_{3}=\mathrm{AALLBB}, \mathrm{G}_{4}=\mathrm{AALVAB}, \mathrm{G}_{5}=\mathrm{AALVBB}$, $\mathrm{G}_{6}=\mathrm{ABLLAA}, \mathrm{G}_{7}=\mathrm{ABLLAB}, \mathrm{G}_{8}=\mathrm{ABLLBB}, \mathrm{G}_{9}=\mathrm{ABLVAA}, \mathrm{G}_{10}=\mathrm{ABLVAB}$ e $\mathrm{G}_{11}=\mathrm{ABLVBB}$ ) were used. Data were obtained from three genetic groups: 1/2Canchim-Nelore (CN), 1/2Angus-Nelore (AN) and 1/2Simmental-Nelore (SN), born in 1998 and 1999 in Southeast Brazil. From five nonlinear models studied: Brody, Von Bertalanffy, Richards, Gompertz and Logistic, the more adequate was the last one. The parameters $A$ (asymptotic value), $k$ (maturing rate) and $m$ (inflection point) estimated by Logistic model for each animal, were analyzed by ordinary least squares, by a linear model that included, besides the overall mean, the fixed effects of the genotype, year of birth, sex and feed management. For the $\mathrm{CN}$ genetic groups, was detected significant effect of genotype for $A$ and $k$ parameters estimates. The genotypes $\mathrm{G}_{3}$ showed inferior value of $A$ and superior of $k$ in relation to $\mathrm{G}_{7}$ and $\mathrm{G}_{8}$. Considering the $\mathrm{AN}$ and $\mathrm{SN}$ genetic groups, the effects of genotype on $A, k$ and $m$ parameters were not significant. Nonlinear model application in this study of association between genetic marker and weight trait in beef cattle provided a fitted analysis of the shape of the growth curve regarding the genotype for the CSN3, GH and LGB genes.
\end{abstract}

Key Words: Bos taurus x Bos indicus, growth curves, ponderal performance, genetic marker, logistic model

\footnotetext{
1 Parte da tese de Doutorado do primeiro autor, apresentada à ESALQ - USP - Piracicaba - SP, financiada pelo CNPq e pela FAPESP. 2 Pesquisador Científico do Instituto de Zootecnia - APTA/SAA e Bolsita de Doutorado do CNPq, ESALQ, USP (ccppaz@aptaregional.sp.gov.br).

3 Professor do Departamento de Zootecnia da Escola Superior de Agricultura "Luiz de Queiroz", ESALQ, USP, Piracicaba, SP.

${ }^{4}$ Pesquisador Embrapa Pecuária Sudeste, São Carlos - SP (ribeiro@cppse.embrapa.br; luciana@cppse.embrapa.br; mauricio@cppse.embrapa.br; geraldo@cppse.embrapa.br).

${ }^{5}$ Bolsista de Doutorado da FAPESP, Universidade Federal de São Carlos - SP.
} 


\section{Introdução}

Os polimorfismos genéticos têm sido associados às características de interesse econômico, como por exemplo o peso em determinada idade do animal. Fatores inerentes a esta área de pesquisa, como o custo de obtenção das análises laboratoriais para determinação destes polimorfismos, têm determinado o pequeno tamanho de amostras destes experimentos. Outro fator relevante são os pesos obtidos ao longo da vida do animal, que são correlacionados entre si, pois os genes que influenciam o peso em determinada idade podem influenciá-lo nas demais idades (pleiotropia), além da estrutura dos dados, que geralmente não é completa, e da variância do peso em função da idade, que é crescente.

Alternativamente, o ajuste de curvas de crescimento, por meio de modelos não-lineares, pode minimizar estes problemas. As funções não-lineares utilizadas para descrever o desenvolvimento dos animais contém informações biológicas relevantes, resumidas em pequeno número de parâmetros. Estas funções podem ainda fornecer informações importantes sobre a variação genética e ambiental que ocorre entre as avaliações consecutivas (Mansour et al., 1991).

Modelos não-lineares como o Brody (Brody, 1945), o Von Bertalanffy (Von Bertalanffy, 1957), o Richards (Richards, 1959), o Logístico (Nelder, 1961) e o Gompertz (Laird, 1965), são os mais empregados em estudos de desenvolvimento animal. Os objetivos principais do ajuste de curvas de crescimento são descrever e predizer o crescimento e fazer inferências com base nas interpretações dos parâmetros (Ratkoswsky, 1983).

O desenvolvimento ponderal dos animais pode ser visto como processo contínuo, em que a taxa de crescimento relativo em função da idade aumenta do nascimento até atingir o ponto em que o crescimento é máximo e, a partir deste ponto, decresce até atingir valores próximos a zero, quando o tamanho máximo do indivíduo é atingido.

A forma da curva de crescimento é muito influenciada pela correlação negativa entre o tamanho adulto e a precocidade (Fitzhugh Jr. \& Taylor, 1971; Fitzhugh Jr., 1976). Dessa forma, acredita-se que melhorias na eficiência do processo de crescimento de bovinos de corte serão possíveis por meio de alterações na taxa de maturação e na composição corporal, independentemente do tamanho adulto, apesar de diferenças serem relatadas quanto à eficiência do processo de crescimento dos animais, além da associação entre esta eficiência e parâmetros da curva de crescimento (Webster, 1989; Huges \& Pitchford, 1994) e das diferenças genéticas entre animais e raças, quanto aos parâmetros da curva de crescimento (Mezzadra \& Miquel, 1994; Oliveira et al., 1994).

Estudos envolvendo a associação entre polimorfismos genéticos e a curva de crescimento são praticamente inexistentes na literatura. Foi encontrado um único relato de associação entre polimorfismos do hormônio do crescimento (GH-AluI e GH-MspI) e os parâmetros da curva de crescimento de bovinos da raça Nelore (Unanian et al., 2000).

No presente estudo, objetivou-se ajustar o modelo Logístico a dados de crescimento de bovinos cruzados $1 / 2$ Canchim-Nelore (CN), 1/2Angus-Nelore (AN) e $1 / 2$ Simental-Nelore $(\mathrm{SN})$ e verificar se os genes da kappa-caseína-HinfI (CSN3), do hormônio do crescimento-AluI (GH) e da $\beta$-lactoglobulina-HaeIII (LGB) influenciam a função de crescimento dos animais dos três cruzamentos estudados.

\section{Material e Métodos}

As 230 observações utilizadas neste estudo foram provenientes de um experimento de avaliação de sistemas de cruzamento entre raças bovinas de corte, pertencentes aos grupos genéticos: $1 / 2$ CanchimNelore (83 animais), $1 / 2$ Angus-Nelore (76 animais) e $1 / 2$ Simental-Nelore (71 animais), nascidos nos anos de 1998 e 1999. Foram utilizados 2, 6 e 4 touros, respectivamente, para as raças Canchim, Angus e Simental, 57 fêmeas Nelore acasaladas com cada uma das duas primeiras raças de touros (Canchim e Angus) e 53 fêmeas Nelore acasaladas com touros da raça Simental. Foram eliminadas 37 observações do arquivo de dados original, restando 65, 69 e 59 animais dos grupos genéticos 1/2Canchim-Nelore, 1/2AngusNelore e $1 / 2$ Simental-Nelore, respectivamente. Dessa forma, foram usadas 193 das 230 observações de animais, tomando-se, em cada animal, 14 mensurações de pesos $(\mathrm{kg})$ ao nascer e ao desmame (7 meses) e pesos mensais dos 8 aos 19 meses de idade. Os animais das raças Canchim, Nelore e cruzados e o sêmen dos touros das raças Angus e Simental pertencem à Embrapa Pecuária Sudeste, São Carlos, SP.

Foram empregados os polimorfismos de restrição dos genes da kappa-caseína-HinfI (CSN3) (Barendse et al., 1997), do hormônio do crescimento-AluI (GH)

R. Bras. Zootec., v.33, n.6, p.1416-1425, 2004 
(Taylor et al., 1998) e da $\beta$-lactoglobulina-HaeIII (LGB) (Barendse et al., 1997), que são do tipo RFLP-PCR, distribuídos nos cromossomos 6, 19 e 11 de bovinos, às distâncias de $103 \mathrm{cM}, 72,7 \mathrm{cMe} 150 \mathrm{cM}$, respectivamente. Foram observadas as classes de genótipos LL e LV para o GH e AA, AB e BB para CSN3 e LGB.

Inicialmente, foram organizadas 22 classes de genótipos identificadas pela concatenação de CSN3, GH e LGB. Entretanto, foram eliminados os animais de genótipos desconhecidos para qualquer um dos genes estudados ou de genótipos que não convergiram na análise realizada pela técnica de modelos não-lineares e usando o PROC NLIN (SAS, 2001), o que resultou em 11 classes de genótipos $\left(\mathrm{G}_{1}=\right.$ AALLAA, $\mathrm{G}_{2}=$ AALLAB, $\mathrm{G}_{3}=$ AALLBB, $\mathrm{G}_{4}=\mathrm{AALVAB}, \mathrm{G}_{5}=\mathrm{AALVBB}, \mathrm{G}_{6}=\mathrm{ABLLAA}$, $\mathrm{G}_{7}=\mathrm{ABLLAB}, \mathrm{G}_{8}=\mathrm{ABLLBB}, \mathrm{G}_{9}=$ ABLVAA, $\mathrm{G}_{10}=A B L V A B$ e $\mathrm{G}_{11}=$ ABLVBB).

Foram ajustados os modelos Brody, Von Bertalanffy, Richards, Logístico e Gompertz (Tabela 1) para estimar o crescimento do animal em função da idade e avaliar a influência dos genótipos dos polimorfismos dos genes da kappa-caseína-Hinfl, do hormônio do crescimento-AluI e $\beta$-lactoglobulina-HaeIII sobre o crescimento. O método utilizado para o ajuste foi o iterativo de Gauss-Newton modificado, descrito por Hartley (1961), por meio da técnica de modelos não-lineares e do procedimento NLIN (SAS, 2001).

A técnica de análise de modelos não-lineares utiliza o processo iterativo, para obtenção das soluções do sistema de equações, pois não é possível obter solução analítica dos parâmetros como no caso de modelos lineares. O processo iterativo requer o fornecimento de valores iniciais aos parâmetros e, a partir desses valores, é calculada a soma de quadrado do resíduo (SQR). Os parâmetros são continuamente modificados até que a SQR seja minimizada. Os valores iniciais foram considerados a partir da média de cada estimativa de parâmetro obtida da literatura. Foi estabelecido o máximo de 100 iterações para que o critério de convergência fosse atingido. A convergência ocorreu sempre que $\left(S Q R_{j-1}-S_{j} R_{j}\right) /\left(S Q R_{j}+10^{-6}\right)$ foi menor que $10^{-8}$, sendo $\mathrm{SQR}_{\mathrm{j}}$ a soma de quadrados residual na j-ésima iteração.

Para escolher o modelo mais adequado, foram consideradas as dificuldades computacionais (número de animais e de genótipos para os quais a convergência foi atingida e a média de iterações para a convergência), a qualidade do ajuste (coeficiente de determinação, quadrado médio do resíduo e médias dos desvios e do quadrado dos desvios) e interpretação biológica dos parâmetros.

No uso dos modelos de regressão linear, a qualidade do ajuste é expressa em termos de coeficiente de determinação $\left(\mathrm{R}^{2}\right)$. Em regressão não-linear, o coeficiente de determinação também pode ser utilizado como parâmetro na avaliação da qualidade do ajuste, mas o $\mathrm{R}^{2}$ não é facilmente definido nestes modelos. Um dos problemas com a definição de $\mathrm{R}^{2}$ é que este requer a presença de intercepto no modelo, parâmetro que nem sempre compõe os modelos não-lineares. De acordo com Schabenberger (2001), uma medida relativamente próxima ao $\mathrm{R}^{2}$, no caso dos modelos não-lineares, utilizada no presente estudo para cálculo de $\mathrm{R}^{2}$ é:

$$
\mathrm{R}^{2}=1-\left(\frac{\mathrm{SQ}_{\text {resíduo }}}{\mathrm{SQ}_{\text {TotalCorrigida }}}\right)
$$

em que $\mathrm{SQ}_{\text {resíduo }}$ é a soma de quadrados do resíduo e $\mathrm{SQ}_{\text {TotalCorrigida, }}$ a soma de quadrados total corrigida para a média (Souza, 1998).

Tabela 1 - Forma geral de cada modelo não-linear utilizado e suas respectivas derivadas Table 1 - Nonlinear models general form and their derivatives

\begin{tabular}{|c|c|c|c|c|}
\hline $\begin{array}{l}\text { Modelo } \\
\text { Model }\end{array}$ & $\begin{array}{l}\text { Forma geral } \\
\text { General form }\end{array}$ & $\begin{array}{l}\mathrm{dy} / \mathrm{dA} \\
d y / d A\end{array}$ & $\begin{array}{l}\mathrm{dy} / \mathrm{db} \\
d y / d b\end{array}$ & $\begin{array}{l}\mathrm{dy} / \mathrm{dk} \\
d y / d k\end{array}$ \\
\hline $\begin{array}{l}\text { Brody } \\
\text { Von Bertalanffy } \\
\text { Richards } 1 \\
\text { Logístico }^{2} \\
\text { Gompertz }\end{array}$ & 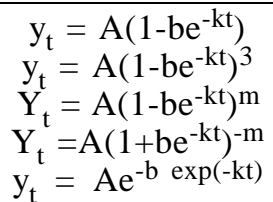 & $\begin{array}{l}\left(1-b e^{-k t}\right) \\
\left(1-b e^{-k t}\right)^{3} \\
\left(1-b e^{-k t}\right)^{m} \\
\left(1+b e^{-k t}\right)^{-m} \\
e^{-b} \exp (-k t)\end{array}$ & $\begin{array}{c}-\mathrm{Ae}^{-\mathrm{kt}} \\
-3 \mathrm{Ae}^{-\mathrm{kt}}\left(1-\mathrm{be} \mathrm{e}^{-\mathrm{kt}}\right)^{2} \\
-\mathrm{Ame}^{-\mathrm{kt}}\left(1-\mathrm{be}^{-\mathrm{kt}}\right)^{(\mathrm{m}-1)} \\
\left.-\mathrm{Ae}^{-\mathrm{kt}} \mathrm{e}^{(-\mathrm{b}} \exp (-\mathrm{kt})\right)\end{array}$ & $\begin{array}{c}\text { Abte }^{-k t} \\
3 \operatorname{Abte}^{-k t}\left(1-b e^{-k t}\right)^{2} \\
\text { Ambte }^{-k t}\left(1-b e^{-k t}\right)^{(m-1)} \\
\text { Amte }{ }^{-k t}\left(1+e^{-k t}\right)^{(-m-1)} \\
\text { Abt } \mathrm{e}^{-k t} e^{-b} \exp (-k t)\end{array}$ \\
\hline
\end{tabular}

${ }^{1} \mathrm{dy} / \mathrm{dm}=\mathrm{A} \ln \left(1-\mathrm{Be}^{-\mathrm{kt}}\right)\left(1-\mathrm{Be}^{-\mathrm{kt}}\right)^{\mathrm{m}} \quad 2 \mathrm{dy} / \mathrm{dm}=-\mathrm{A} \ln \left(1+\mathrm{e}^{-\mathrm{kt}}\right)\left(1+\mathrm{e}^{-\mathrm{kt}}\right)^{-\mathrm{m}}$

Adaptado de Fitzhugh Jr. (1976) e Perotto et al. (1992).

Adapted from Fitzhugh Jr. (1976) and Perotto et al. (1992).

R. Bras. Zootec., v.33, n.6, p.1416-1425, 2004 
Análises iniciais comprovaram que o modelo Logístico (Nelder, 1961) foi mais apropriado para descrever o crescimento dos animais:

$$
\mathrm{y}_{\mathrm{t}}=\mathrm{A}\left(1+\mathrm{be}^{-\mathrm{kt}}\right)^{-\mathrm{m}}+\varepsilon
$$

em que: $y_{t}$ representa o peso corporal $(\mathrm{kg})$ no tempo $\mathrm{t}$, expresso em dias após o nascimento; $A$, o valor ou peso assintótico; $b$, a constante de integração, que, no modelo Logístico assume o valor $1 ; k$, a taxa de maturidade pós-natal - um indicador da velocidade com que o animal atinge o valor assintótico; $m$, a constante que define a forma da curva nos modelos $\mathrm{e}$, conseqüentemente, o ponto de inflexão da curva; e $\varepsilon$, o erro aleatório.

\section{Resultados e Discussão}

Considerando-se o critério de qualidade de ajuste do modelo (coeficiente de determinação, quadrado médio do resíduo, número de animais que atingiram a convergência e interpretação biológica dos parâmetros), foram eliminados os animais pertencentes às classes de genótipos que não apresentaram ajuste adequado, para o modelo Logístico. Os animais foram representados nos grupos genéticos e nas classes de genótipos da seguinte maneira: 65 animais $\mathbf{C N}$ pertencentes aos genótipos: $\mathrm{G}_{2}=\mathrm{AALLAB} \quad(\mathrm{n}=12), \mathrm{G}_{3}=\mathrm{AALLBB} \quad(\mathrm{n}=33)$, $\mathrm{G}_{7}=\operatorname{ABLLAB}(\mathrm{n}=10), \mathrm{G}_{8}=\operatorname{ABLLBB} \quad(\mathrm{n}=7)$ e $\mathrm{G}_{9}=\operatorname{ABLVAA}(\mathrm{n}=3), 69$ animais $\mathbf{A N}$ das classes de genótipos: $\mathrm{G}_{1}=$ AALLAA $(\mathrm{n}=3), \mathrm{G}_{2}=$ AALLAB $(n=15), G_{3}=\operatorname{AALLBB}(n=14), G_{4}=\operatorname{AALVAB}(n=3)$, $\mathrm{G}_{5}=\mathrm{AALVBB} \quad(\mathrm{n}=8), \mathrm{G}_{6}=$ ABLLAA $\quad(\mathrm{n}=8)$, $\mathrm{G}_{7}=\operatorname{ABLLAB}(\mathrm{n}=7), \mathrm{G}_{10}=\mathrm{ABLVAB}(\mathrm{n}=6)$ e $\mathrm{G}_{11}=\operatorname{ABLVBB}(\mathrm{n}=5)$ e 59 animais $\mathbf{S N}$ das classes de genótipos: $\mathrm{G}_{2}=\mathrm{AALLAB}(\mathrm{n}=8), \mathrm{G}_{3}=\mathrm{AALLBB}$ $(n=26), G_{4}=\operatorname{AALVAB}(n=11), \mathrm{G}_{5}=\operatorname{AALVBB}(\mathrm{n}=5)$, $\mathrm{G}_{8}=\operatorname{ABLLBB}(\mathrm{n}=6)$ e $\mathrm{G}_{11}=\operatorname{ABLVBB}(\mathrm{n}=3)$, para os genes CSN3, GH e LGB respectivamente.

A amplitude dos valores das idades de cada pesagem foi: 146-253, 175-282, 203-311, 238-354, 269-394, 297-425, 325-454, 353-480, 381-512, 409$531,437-549,464-568$ e 495-603 dias, respectivamente, para $\mathrm{P}_{\mathrm{D}}, \mathrm{P}_{8}, \mathrm{P}_{9}, \mathrm{P}_{10}, \mathrm{P}_{11}, \mathrm{P}_{12}, \mathrm{P}_{13}$, $\mathrm{P}_{14}, \mathrm{P}_{15}, \mathrm{P}_{16}, \mathrm{P}_{17}, \mathrm{P}_{18}$ e $\mathrm{P}_{19}$ (pesos mensurados mensalmente dos 7 aos 19 meses de idade, em média).

Considerando-se a dificuldade de realização das análises, o modelo Logístico foi o mais adequado pois foi o que convergiu para o maior número de animais e genótipos. Provavelmente, o fato de os animais ainda não terem atingido a maturidade quando foram realizadas às últimas mensurações dos pesos (19 meses de idade em média), associado à baixa frequiência das pesagens nas fases iniciais da vida (peso medido ao nascimento e depois ao desmame por volta dos 7 meses de idade), pode estar ligado à dificuldade de ajuste dos modelos Brody, Von Bertallanffy, Richards e Gompertz, que não convergiram. Tais resultados podem ser explicados por Nadarajah et al. (1984), que afirmam que, em raças menos precoces, como é o caso das zebuínas, é possível que os modelos com ponto de inflexão variável (Richards e Logístico) sejam mais adequados. Além de que, a alta correlação negativa entre os parâmetros $m$ e $b$ no modelo Richards, geralmente, dificulta o ajustamento deste modelo (Brown et al., 1976). Em estudos mais recentes, Freitas et al. (1998a; 1998b) compararam os modelos Gompertz, Logístico e Von Bertalanffy, utilizando dados de animais da raça Canchim, e concluíram que os doisúltimos proporcionaram boa qualidade de ajuste aos pesos observados, para ambos os sexos, com certa superioridade do modelo Logístico em relação aos demais.

Na Tabela 2, são apresentados os valores médios dos parâmetros da curva de crescimento, estimados pelo modelo Logístico, e o número de animais dos grupos genéticos $\mathrm{CN}, \mathrm{AN}$ e $\mathrm{SN}$ para o qual o critério de convergência foi atingido. Neste modelo, a variação de $m$ é limitada entre 2 e 4 pela fixação de $b=1$ (Brown et al., 1976). Os valores de $m$ praticamente não variaram (variação de 3,51 a 3,57 ).

Com relação à qualidade do ajuste do modelo, que se baseou no coeficiente de determinação $\left(\mathrm{R}^{2}\right)$, no número de animais que atingiram a convergência $(\mathrm{N})$ e na interpretação biológica dos parâmetros, o modelo Logístico pode ser utilizado para descrever o crescimento dos animais das três composições genéticas em estudo.

Os modelos ajustados para os conjuntos de dados (Tabela 2) apresentaram boa qualidade de ajuste, com coeficiente de determinação superior a $79,5 \%$. As proporções de animais que atingiram a convergência (considerando-se os genótipos) foram de 78,$3 ; 90,8$ e $83,1 \%$ respectivamente, para os grupos genéticos $\mathrm{CN}$, AN e $\mathrm{SN}$, corroborando os resultados obtidos por Freitas et al. (1995; 1997; 1998a; 1998b), em que a função Logística apresentou

R. Bras. Zootec., v.33, n.6, p.1416-1425, 2004 
boa qualidade de ajuste aos dados de animais das raças Holandesa e Canchim. Para os grupos genéticos $\mathrm{AN}$ e $\mathrm{SN}$ as estimativas do peso assintótico $(A)$ foram superiores à do $\mathrm{CN}$.

Na Figura 1, estão representadas as curvas do ganho de peso e do peso, em função da idade, ajustadas pelo modelo Logístico, para animais cruzados CN, AN e SN.

O peso assintótico $(A)$ e o índice de maturação $(k)$ são considerados os dois parâmetros de maior importância, sob o ponto de vista zootécnico, principalmente porque fêmeas mais pesadas geralmente criam bezerros que crescem mais rapidamente - o que é desejável em gado de corte. Por outro lado, o acréscimo em gastos para manutenção de vacas de maior porte pode não compensar a vantagem do crescimento mais rápido dos bezerros (Cartwright, 1970).

Oliveira (1995) ajustou cinco modelos objetivando descrever o crescimento de 556 fêmeas da raça Guzerá. No modelo de Von Bertalanffy, que apresentou ajuste mais adequado (de acordo com os critérios adotados), foram estimados valores de $453,9 \mathrm{~kg}$ e 0,06936 mês $^{-1}$ para os parâmetros $A$ e $k$, respectivamente. Apenas para fim de comparação com o presente estudo, no modelo Logístico foram estimados para os parâmetros $A, k$ e $m$ os valores de $445,9 \mathrm{~kg}, 0,090459$ mês $^{-1}$ e 2,98837, respectivamente. Perotto et al., (1997) usaram o modelo Richards para descrever o crescimento de 53 fêmeas da raça Guzerá e encontraram $441,4 \mathrm{~kg}, 0,06513$ mês $^{-1}$ e 3,8416 para as estimativas de $A, k$ e $m$, respectivamente.
Considerando as estimativas médias obtidas para os dados de pesos, 427,5 kg, 475,0 kg e 489,1 $\mathrm{kg}$ para o peso assintótico $(A), 0,00660 \mathrm{dia}^{-1}$, $0,00624 \mathrm{dia}^{-1}$ e $0,00599 \mathrm{dia}^{-1}$ para a taxa de maturação $(k)$ e $3,5710,3,5137$ e 3,5401 para o ponto de inflexão $(m)$, respectivamente, para os grupos genéticos $\mathrm{CN}$, AN e $\mathrm{SN}$, observa-se razoável discordância entre as estimativas dos parâmetros de crescimento obtidas para os animais cruzados e aquelas estimadas para os animais da raça Guzerá (Oliveira, 1995; Perotto et al., 1997), sobretudo quanto ao parâmetro $k$. Como $m$ e $k$ são correlacionados positivamente enquanto a correlação entre $k$ e $A$ é negativa, é possível que o modelo Logístico ( $b=1$ limitando os valores de $m$ entre 2 e 4) limite os valores de $A$.

As curvas ajustadas pelo modelo Logístico, cujas estimativas dos parâmetros estão apresentadas na Tabela 2, que descrevem o crescimento dos animais $\mathrm{CN}$, AN e SN, encontram-se na Figura 2.

Para os dados originais do peso, nota-se que, enquanto o valor do parâmetro $A$ do $\mathrm{CN}$ foi $10 \%$ e $12,5 \%$ inferior ao valor do mesmo parâmetro para o AN e SN, respectivamente, o valor de $A$ para o AN foi de apenas 3\% inferior em relação ao $\mathrm{SN}$. O valor de $k$ do $\mathrm{CN}$ foi $5 \%$ superior ao do AN e 9\% superior ao do SN; para AN foi $4 \%$ superior ao do SN. Estes resultados corroboram a sugestão de que certos cruzamentos podem contornar parcialmente o antagonismo biológico entre peso adulto e taxa de maturação em peso existente dentro de raças (Fitzhugh Jr. \& Taylor, 1971).

Tabela 2 - Estimativas dos parâmetros $(A, k$ e $m)$ e respectivos erros-padrão, número de animais (N), coeficiente de determinação $\left(\mathrm{R}^{2}\right)$ e número de iterações $(\mathrm{NI})$ dos modelos ajustados para os dados de peso de bovinos cruzados

Table 2 - Parameter estimates $(A, k$ and $m)$, stander error, number of animals $(N)$, coefficient of determination $\left(R^{2}\right)$ and number of iteration (NI) of the adjusted models from data of the body weight (W) from the three genetic groups

\begin{tabular}{|c|c|c|c|c|c|}
\hline $\begin{array}{l}\text { Grupo genético } \\
\text { Genetic group }\end{array}$ & $\begin{array}{c}A(\mathrm{~kg}) \pm \text { erro-padrão } \\
A(\mathrm{~kg}) \pm \text { standard error }\end{array}$ & $\begin{array}{l}k\left(\text { dia }^{-1}\right) \pm \text { erro-padrão } \\
k\left(\text { day }^{-1}\right) \pm \text { standard error }\end{array}$ & $\begin{array}{c}m \pm \text { erro-padrão } \\
m \pm \text { standard error }\end{array}$ & $\begin{array}{l}\mathrm{R}^{2}(\%) \\
R^{2}(\%)\end{array}$ & $\begin{array}{l}\mathrm{NI} \\
N I\end{array}$ \\
\hline $\begin{array}{c}1 / 2 \text { Canchim-Nelore } \\
1 / 2 \text { Canchim-Nellore } \\
(\mathrm{N}=65)\end{array}$ & $427,5 \pm 10,3$ & $0,00660 \pm 0,00037$ & $3,5710 \pm 0,1959$ & 79,5 & 5 \\
\hline $\begin{array}{c}1 / 2 \text { Angus-Nelore }{ }^{1 / 2} \\
\text { Angus-Nellore } \\
(\mathrm{N}=69)\end{array}$ & $475,0 \pm 10,1$ & $0,00624 \pm 0,000285$ & $3,5137 \pm 0,1442$ & 84,5 & 4 \\
\hline $\begin{array}{c}1 / 2 \text { Simental-Nelore } \\
1 / 2 \text { Simmental-Nellore } \\
(\mathrm{N}=59)\end{array}$ & $489,1 \pm 13,8$ & $0,00599 \pm 0,000346$ & $3,5401 \pm 0,1699$ & 83,7 & 4 \\
\hline
\end{tabular}

R. Bras. Zootec., v.33, n.6, p.1416-1425, 2004 

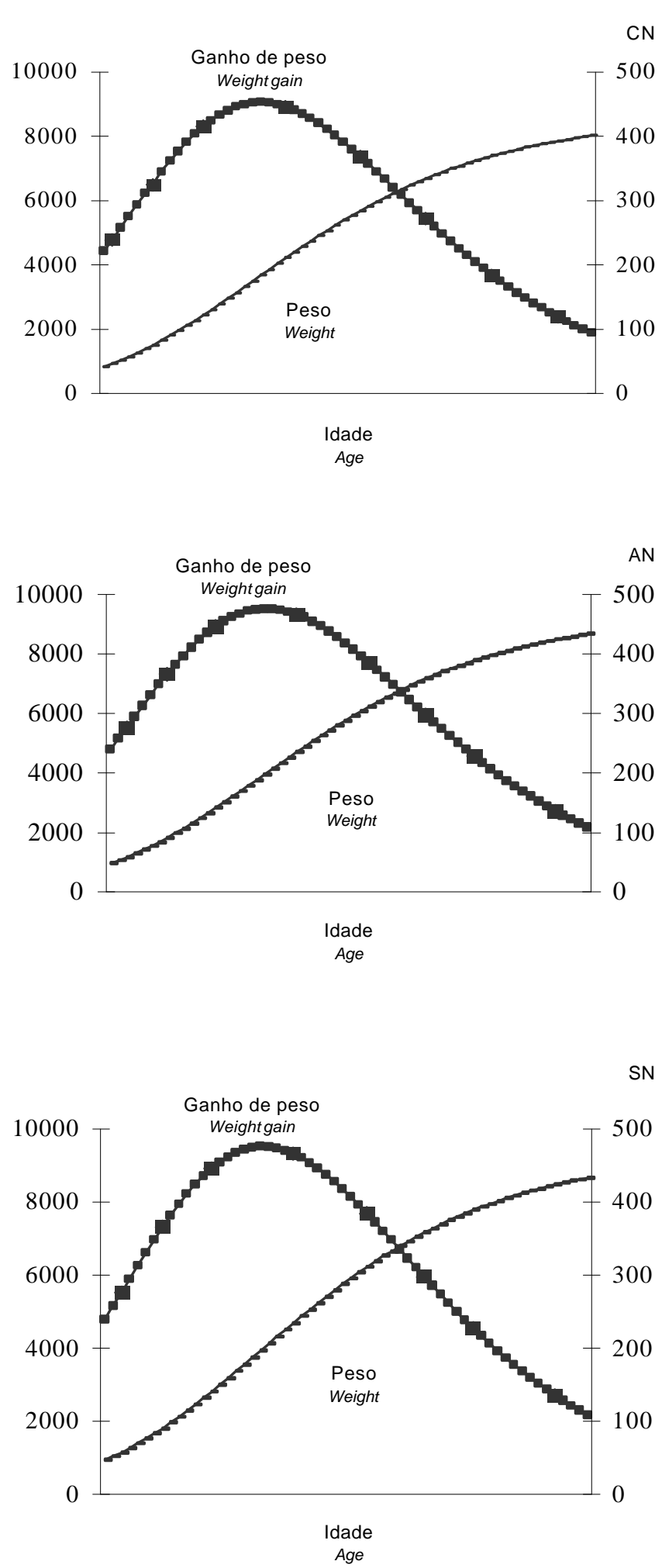

Figura 1 - Estimativa do ganho de peso (g/10dias) e do peso $(\mathrm{kg})$, em função da idade (dias), ajustado pelo modelo Logístico, para os grupos genéticos: $1 / 2$ Canchim-Nelore (CN), 1/2AngusNelore (AN) e $1 / 2$ Simental-Nelore (SN).

Figure 1 - Estimate of weight gain (g/10days) and body weight $(\mathrm{kg})$ in accordance with age (days), adjusted by Logistic model, from the genetic groups: $1 / 2$ CanchimNellore (CN), 1/2Angus-Nellore (AN) e 1/2SimmentalNellore (SN).
Constam, na Tabela 3, as estimativas dos parâmetros $(A, k$ e $m)$, pelo modelo Logístico, o coeficiente de determinação, o número de animais por genótipo que atingiram a convergência e o número de iterações dos modelos de cada classe de genótipo, de animais cruzados $\mathrm{CN}$, AN e $\mathrm{SN}$.

Em geral, o modelo Logístico proporcionou boa qualidade de ajuste, com $\mathrm{R}^{2}$ variando de 73,5 a $98,5 \%$. O peso assintótico também apresentou valores razoáveis, com exceção dos animais $A N$ do genótipo $\mathrm{G}_{11}$ (ABLVBB), cuja estimativa de $A$ foi superior a $700 \mathrm{~kg}$.

As estimativas do parâmetros $A, k$ e $m$ obtidas para cada animal, após transformadas pelo logaritmo natural, foram analisadas pela técnica de modelos lineares usando o PROC GLM (SAS, 2001), a fim de se testar o efeito do genótipo e para o estudo de contrastes. Os modelos utilizados incluíram os efeitos fixos de ano de nascimento, sexo, manejo alimentar e genótipo. $\mathrm{O}$ modelo linear descrito acima foi utilizado para os três conjunto de dados (grupos genéticos $\mathrm{CN}$, AN e SN).

A transformação logarítmica foi usada, uma vez que não foi atendida uma das suposições (linearidade) feitas em análises usando a técnica de modelos lineares. Estas análises especificam que a média condicional $\mathrm{E}\left(\mathrm{y} \mid \mathrm{x}=\mathrm{x}_{0}\right)$ da variável resposta $\mathrm{y}$, dado o valor $\mathrm{x}_{0}$ do vetor preditor $\mathrm{x}$, é linear em $\mathrm{x}_{0}$. A aplicação de modelos lineares pode ser estendida supondo-se que uma transformação apropriada da variável resposta dada por $\mathrm{t}(\mathrm{y})$, em que $\mathrm{E}\{\mathrm{t}(\mathrm{y}) \mid \mathrm{x}\}$, seja linear em $\mathrm{x}$, na função $\mathrm{t}(\mathrm{y})=\beta_{0}+\beta^{\mathrm{T}} \mathrm{x}+\varepsilon$, para $\beta_{0}$ e $\beta^{\mathrm{T}}$ desconhecidos. O termo $\varepsilon$ (erro aleatório) é independente de $\mathrm{x}$ e tem média zero (Cook \& Weisberg, 1994).

Não foi observado efeito significativo $(\mathrm{P}>0,05)$ do genótipo sobre os três parâmetros $(A, k$ e $m)$ do modelo Logístico para os conjuntos de dados AN e SN. Os coeficientes de variação foram 5,45 e - $5,97 \%$ e os valores de $\mathrm{R}^{2}, 51,88$ e $33,07 \%$, respectivamente, para $A$ e $k$ (transformados para o logaritmo natural) na análise realizada com os animais AN. Para o grupo genético $\mathrm{SN}$, os coeficientes de variação foram 4,95 e $-6,77 \%$ e os valores de $\mathrm{R}^{2}, 48,63$ e $18,48 \%$, respectivamente, para $A$ e $k$ (transformados para o logaritmo natural).

Para os animais do grupo genético $\mathrm{CN}$, os genótipos influenciaram significativamente as estimativas dos parâmetros $A$ e $k(\mathrm{P}<0,01$ e $\mathrm{P}<0,05$, respectivamente), porém não influenciaram $(\mathrm{P}>0,05)$ o parâmetro $m$. Os coeficientes de variação foram

R. Bras. Zootec., v.33, n.6, p.1416-1425, 2004 
3,83 e $-4,90 \%$ e os valores de $\mathrm{R}^{2}, 75,5$ e $57,81 \%$, respectivamente, para $A$ e $k$ (transformados para o logaritmo natural). $\mathrm{O}$ genótipo $\mathrm{G}_{3}$ apresentou valor inferior para o peso assintótico $(A)$ e maior taxa de maturação por unidade de tempo $(k)$, em relação aos genótipos $\mathrm{G}_{7}$ e $\mathrm{G}_{8}$. No estudo de contrastes, compararam-se o genótipo $\mathrm{G}_{3}$ com os genótipos $\mathrm{G}_{7} \mathrm{e}$ $\mathrm{G}_{8}$ e observaram-se diferenças significativas $(\mathrm{P}<0,001)$ tanto para $A$ quanto para $k$, indicando influência do gene CSN3 sobre estes parâmetros. Os valores do parâmetro $A$ para o genótipo $\mathrm{G}_{3}$ foram 6,5 e $14,5 \%$ inferiores ao mesmo parâmetro para os genótipos $\mathrm{G}_{7}$ e $\mathrm{G}_{8}$, respectivamente, e as diferenças quanto à taxa de maturação $(k)$ foram 14 e $21 \%$ superiores para $G_{3}$ em relação aos genótipos $\mathrm{G}_{7}$ e $\mathrm{G}_{8}$. Provavelmente, o alelo A do gene CSN3 exerça influência favorável sobre os parâmetros $A$ e $k$. Apesar de não-significativa, a diferença entre $\mathrm{G}_{2} \mathrm{eG}_{7}$, o valor de $A$ para o genótipo $\mathrm{G}_{2}$ foi $4 \%$ inferior em relação ao $\mathrm{G}_{7}$, e de $k, 10 \%$ superior para $\mathrm{G}_{2}$ em relação ao $\mathrm{G}_{7}$, indicando tendência de o alelo B do gene LGB apresentar efeito favorável no crescimento animal.

Estas diferenças são descritas visualizadas na Figura 3, que apresenta as estimativas dos pesos, em função da idade, para cada genótipo $\left(\mathrm{G}_{1}, \mathrm{G}_{2}\right.$, $\mathrm{G}_{3}, \mathrm{G}_{4}, \mathrm{G}_{5}, \mathrm{G}_{6}, \mathrm{G}_{7}, \mathrm{G}_{8}, \mathrm{G}_{9}, \mathrm{G}_{10}$ e $\mathrm{G}_{11}$ ), formado pelos genes CSN3, GH e LGB, de animais cruzados $\mathrm{CN}, \mathrm{AN}$ e SN.

Mesmo o genótipo não tendo apresentado efeito significativo sobre os parâmetros da curva de crescimento dos grupos genéticos $\mathrm{AN}$ e $\mathrm{SN}$, usando o modelo de análise proposto, foram observadas algumas diferenças entres as classes de genótipos. Em geral, houve tendência de os genes CSN3 e LGB apresentarem efeito sobre os parâmetros $A$ e $k$, conforme descrito anteriormente. Lin et al. (1987) encontraram associação de CSN3 e LGB com peso ao nascimento, peso aos 350 dias de idade e com peso ao primeiro parto, e Moody et al. (1996) encontraram associação de CSN3 com características de

Tabela 3 - Estimativas dos parâmetros $(A, k$ e $m)$, número de iterações $(\mathrm{NI})$, coeficiente de determinação $\left(R^{2}\right)$, número de animais por grupo genético $(N)$ e genotípico $(N G)$, que atingiram a convergência para os modelos Logísticos ajustados para dados de peso em função da idade de cada grupo genotípico de bovinos de três grupos genéticos

Table 3 - Parameter estimates $(A, k$ and $m)$, number of iteration $(\mathrm{NI})$, coefficient of determination $\left(R^{2}\right)$ number of animals by genetic group (N) and by genetic group (NG) of the adjusted Logistics models from data of the body weight, according to the age, for genotype groups from the three genetic groups

\begin{tabular}{|c|c|c|c|c|c|}
\hline $\begin{array}{l}\text { Grupo genético } \\
\text { Genetic group }\end{array}$ & $\begin{array}{c}\text { Grupo genotípico }{ }^{1} \\
\text { Genotype group }\end{array}$ & $A(\mathrm{~kg})$ & $k\left(\right.$ dia $\left.^{-1}\right)$ & $m$ & $\mathrm{R}^{2}(\%)$ \\
\hline \multirow{5}{*}{$\begin{array}{c}1 / 2 \text { Canchim-Nelore } \\
1 / 2 \text { Canchim-Nellore } \\
(\mathrm{N}=65)\end{array}$} & $\mathrm{G} 2(\mathrm{NG}=12)$ & $429,6(\mathrm{NI}=5)$ & 0,00661 & 3,7508 & 84,9 \\
\hline & $\mathrm{G} 3(\mathrm{NG}=33)$ & $417,7(\mathrm{NI}=5)$ & 0,00697 & 3,6771 & 81,3 \\
\hline & $\mathrm{G} 7(\mathrm{NG}=10)$ & $446,8(\mathrm{NI}=5)$ & 0,00599 & 3,3909 & 74,4 \\
\hline & $\mathrm{G} 8(\mathrm{NG}=7)$ & $488,9(\mathrm{NI}=5)$ & 0,00551 & 3,2604 & 73,5 \\
\hline & G9 $(\mathrm{NG}=3)$ & $369,0(\mathrm{NI}=7)$ & 0,00716 & 3,4876 & 84,9 \\
\hline \multirow{9}{*}{$\begin{array}{c}\text { 1/2Angus-Nelore } \\
\text { 1/2Angus-Nellore } \\
(\mathrm{N}=69)\end{array}$} & $\mathrm{G} 1(\mathrm{NG}=3)$ & $429,9(\mathrm{NI}=5)$ & 0,00591 & 3,1549 & 86,4 \\
\hline & $\mathrm{G} 2(\mathrm{NG}=15)$ & $462,3(\mathrm{NI}=5)$ & 0,00598 & 3,3647 & 84,4 \\
\hline & $\mathrm{G} 3(\mathrm{NG}=14)$ & $492,2(\mathrm{NI}=5)$ & 0,00604 & 3,3728 & 88,0 \\
\hline & $\mathrm{G} 4(\mathrm{NG}=3)$ & $468,0(\mathrm{NI}=8)$ & 0,00741 & 4,2694 & 97,3 \\
\hline & G5 $(\mathrm{NG}=8)$ & $454,8(\mathrm{NI}=6)$ & 0,00714 & 4,0263 & 88,8 \\
\hline & G6 $(\mathrm{NG}=8)$ & $468,7(\mathrm{NI}=5)$ & 0,00669 & 3,5436 & 84,4 \\
\hline & G7 $(\mathrm{NG}=7)$ & $471,9(\mathrm{NI}=5)$ & 0,00567 & 3,2044 & 80,8 \\
\hline & $\mathrm{G} 10(\mathrm{NG}=6)$ & $560,4(\mathrm{NI}=10)$ & 0,00591 & 3,9079 & 93,1 \\
\hline & $\mathrm{G} 11(\mathrm{NG}=5)$ & $795,3(\mathrm{NI}=6)$ & 0,00397 & 3,9085 & 94,1 \\
\hline \multirow{6}{*}{$\begin{array}{c}1 / 2 \text { Simental-Nelore } \\
1 / 2 \text { Simmental-Nellore } \\
(\mathrm{N}=59)\end{array}$} & $\mathrm{G} 2(\mathrm{NG}=8)$ & $538,9(\mathrm{NI}=5)$ & 0,00519 & 3,5562 & 77,9 \\
\hline & $\mathrm{G} 3(\mathrm{NG}=26)$ & $485,4(\mathrm{NI}=5)$ & 0,00608 & 3,5461 & 84,6 \\
\hline & $\mathrm{G} 4(\mathrm{NG}=11)$ & $508,1(\mathrm{NI}=5)$ & 0,00628 & 3,8055 & 85,7 \\
\hline & G5 $(\mathrm{NG}=5)$ & $472,6(\mathrm{NI}=4)$ & 0,00599 & 3,5425 & 78,6 \\
\hline & $\mathrm{G} 8(\mathrm{NG}=6)$ & $512,5(\mathrm{NI}=4)$ & 0,00567 & 3,1427 & 84,3 \\
\hline & $\mathrm{G} 11(\mathrm{NG}=3)$ & $429,0(\mathrm{NI}=5)$ & 0,00694 & 3,5319 & 98,6 \\
\hline
\end{tabular}

${ }^{1} G_{1}=A A L L A A ; G_{2}=A A L L A B ; G_{3}=A A L L B B ; G_{4}=A A L V A B ; G_{5}=A A L V B B ; G_{6}=A B L L A A ; G_{7}=A B L L A B ;$ $\mathrm{G}_{8}=A B L L B B ; \mathrm{G}_{9}=A B L V A A ; \mathrm{G}_{10}=A B L V A B ; \mathrm{G}_{11}=A B L V B B$.

R. Bras. Zootec., v.33, n.6, p.1416-1425, 2004 
crescimento (valor genético do peso ao nascimento, ganho do nascimento ao desmame, ganho do desmame ao ano e valor genético materno do peso ao nascimento e ganho do nascimento ao desmame).

A tendência de efeito do polimorfismo do GH (grupo genético SN) sobre os parâmetros da curva de crescimento está de acordo com resultados da literatura (Rocha et al., 1992; Unaniam et al., 2000; Tambasco et al., 2003), que sugerem possível associação de GH com características de crescimento em bovinos de corte.

Nas análises de características quantitativas usando o modelo infinitesimal, a interação entre locos (epistasia) não é considerada em razão da dificuldade de análises com o uso de modelos matemáticos que possam estimar este efeito, além de dificuldades computacionais. Outro fator que justifica a não-inclusão do efeito de epistasia nos modelos é a falta de evidências de que este efeito possa influenciar as características quantitativas. Entretanto, recentemente a interação entre locos (epistasia) têm sido relatada na literatura (Casas et al., 2000; Tambasco et al., 2003).

Pode estar ocorrendo interação entre os locos dos genes estudados ou, ainda, com locos de outros genes que estão muito próximos à localização destes, e, portanto, estão segregando ligados. Em geral, os

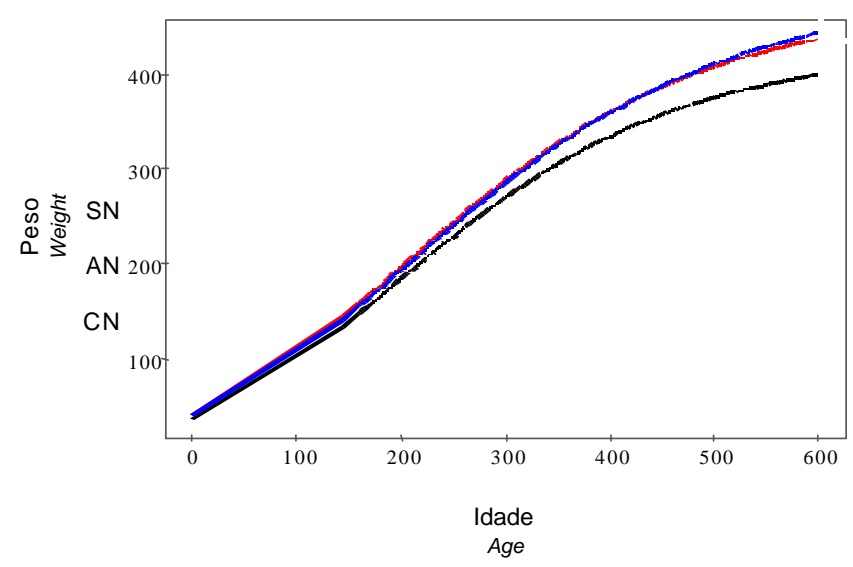

Figura 2 - Estimativa do peso $(\mathrm{kg})$, em função da idade (dias), ajustada pelo modelo Logístico para animais dos grupos genéticos: $1 / 2$ CanchimNelore (CN), $1 / 2$ Angus-Nelore (AN) e $1 / 2$ Simental-Nelore (SN).

Figure 2 - Estimate of body weight (kg), according to the age (days), adjusted by Logistic model from genetic groups: $1 / 2$ Canchim-Nellore (CN), 1/2Angus-Nellore (AN) e $1 / 2$ Simmental-Nellore (SN).
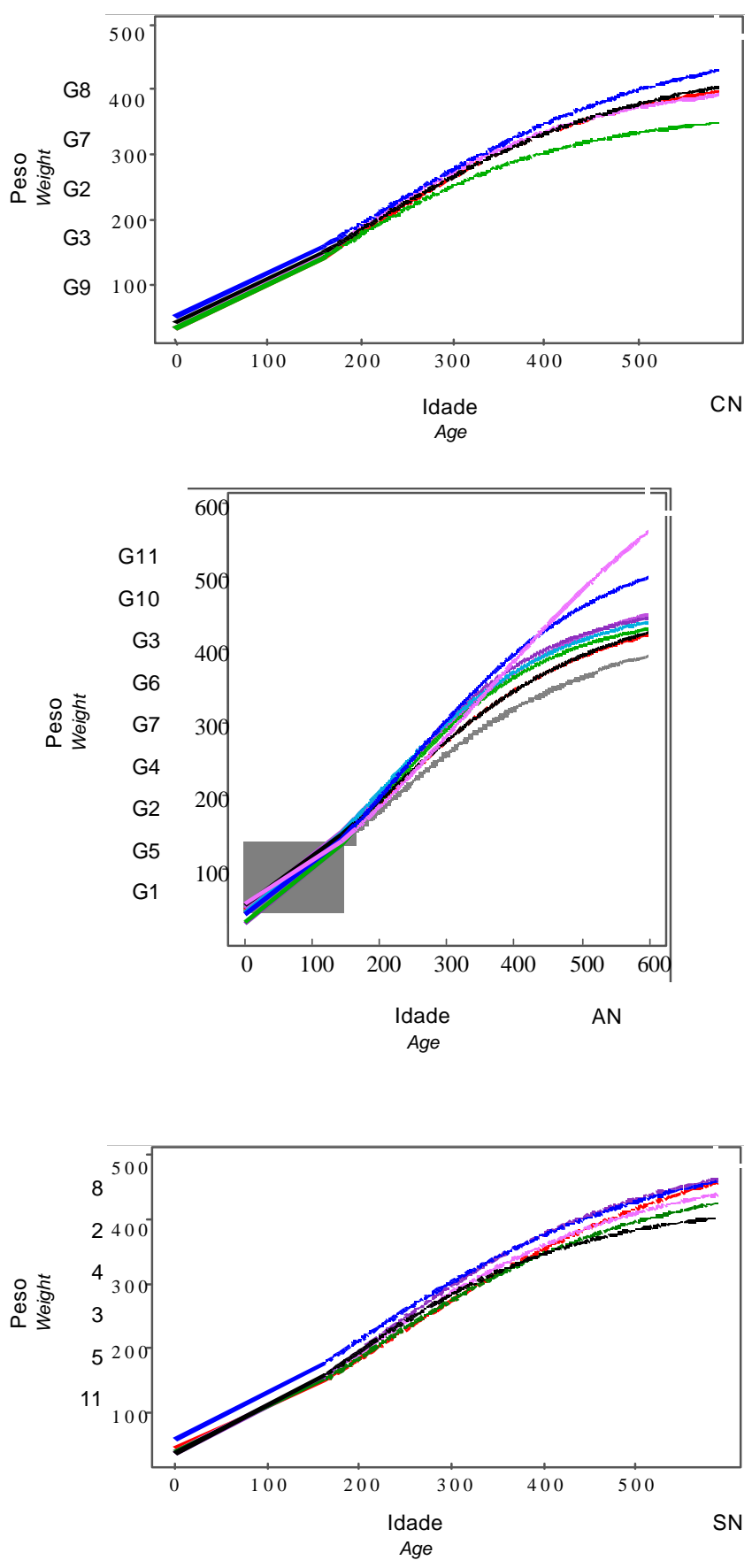

$\mathrm{G}_{1}=\mathrm{AALLAA} ; \mathrm{G}_{2}=\mathrm{AALLAB} ; \mathrm{G}_{3}=\mathrm{AALLBB} ; \mathrm{G}_{4}=\mathrm{AALVAB}$; $\mathrm{G}_{5}=\mathrm{AALVBB} ; \mathrm{G}_{6}=$ ABLLAA; $\mathrm{G}_{7}=\mathrm{ABLLAB} ; \mathrm{G}_{8}=\mathrm{ABLLBB} ;$ $\mathrm{G}_{9}=A B L V A A ; \mathrm{G}_{10}=A B L V A B ; \mathrm{e}_{11}=A B L V B B$.

Figura 3 - Estimativa do peso (kg), em função da idade (dias), ajustada pelo modelo Logístico para os grupos genotípicos de bovinos dos grupos genéticos: $1 / 2$ Canchim-Nelore (CN), 1/2AngusNelore (AN) e $1 / 2$ Simental-Nelore (SN).

Figure 3 - Estimate of body weight ( $\mathrm{kg})$, according to the age (days), adjusted by Logistic model for the genotype groups from the genetic groups: $1 / 2$ Canchim-Nellore (CN), $1 / 2$ Angus-Nellore (AN) and $1 / 2$ SimmentalNellore (SN).

R. Bras. Zootec., v.33, n.6, p.1416-1425, 2004 
genótipos $\mathrm{AB}$ e BB para LGB apresentaram forma da curva de crescimento mais desejável que os genótipos AA, na presença do genótipo LL para GH, e o genótipo BB para LGB apresentou forma da curva mais desejável que o $\mathrm{AB}$, na presença do genótipo LV para $\mathrm{GH}$, em conformidade com os resultados de Tambasco et al. (2003), que encontraram evidências de interação entre GH e LGB e associação de GH com ganho de peso do nascimento ao desmame e do desmame aos 12 meses de idade, em animais cruzados. Os genótipos AB e BB para LGB na presença de LL para $\mathrm{GH}$, apresentaram maior ganho de peso que o genótipo AA, enquanto o genótipo AA, maior ganho de peso que o $\mathrm{BB}$ na presença de $\mathrm{LV}$ para GH.

A aplicação da técnica de modelos não-lineares em estudos de associação entre polimorfismos genéticos e o crescimento animal proporcionou análise detalhada do desenvolvimento dos animais dos diferentes genótipos (genes: CSN3, GH e LGB). Entretanto, mais estudos são necessários para confirmar a possível associação destes e de outros genes com o crescimento de bovinos de corte e as possíveis interações entre genes.

\section{Conclusões}

O modelo Logístico forneceu adequada estimação da curva de crescimento para os polimorfismos dos genes kappa-caseína (CSN3), hormônio do crescimento (GH) e $\beta$-Lactoglobulina (LGB) de animais dos grupos genéticos: $1 / 2$ Canchim-Nelore $(\mathrm{CN})+1 / 2$ Angus-Nelore (AN) e $1 / 2$ Simental-Nelore (SN).

Foram constatados efeitos dos polimorfismos dos genes da kappa-caseína-HinfI (CSN3), do hormônio do crescimento-AluI e da $\beta$-lactoglobulina-HaeIII (LGB) sobre as estimativas do parâmetros $A, k$ e $m$ do modelo Logístico usado para descrever o crescimento de bovinos cruzados.

\section{Literatura Citada}

BARENDSE, W.; VAIMAN, D.; KEMP, S.J. et al. A mediumdensity genetic linkage map of the bovine genome. Mammalian Genome, v.8, p.21-28, 1997.

BRODY, S. Bioenergetics and growth. New York: Reinhold Publishing Corp., 1945. 1023p.

BROWN, J.E.; FITZHUGH JR., H.A.; CARTWRIGHT, T.C.A. A comparision of nonlinear models for describing weight-age relationships in cattle. Journal Animal Science, v.42, p.810-818, 1976.
CARTWRIGHT, T.C. Selection criteria for beef cattle for the future. Journal of Animal Science, v.30, p.706-711, 1970.

CASAS, E.; SHACKELFORD, S.D.; KEELE, J.W. et al. Quantitative trait loci affecting growth and carcass composition of cattle segregating alternate forms of myostatin. Journal of Animal Science, v.78, p.560-569, 2000.

COOK, R.D.; WEISBERG, S. Transforming a response variable for linearity. Biometrika, v.81, n.4, p.731-737, 1994.

FITZHUGH JUNIOR, H.A. Analysis of growth curves and strategies for altering their shape. Journal of Animal Science, v.42, p.1036-1051, 1976.

FITZHUGH JR., H.A. Genetic analysis of degree of maturity. Journal of Animal Science, v.33, n.4, p.717-725, 1971.

FREITAS, A.F.; ALENCAR, M.M.; SILVA, A.S. Ajuste de modelos não lineares em bovinos de corte. I. Padrão da população. In: REUNIÃO ANUAL DA SOCIEDADE BRASILEIRA DE ZOOTECNIA, 35., 1998, Botucatu. Anais... Botucatu: Sociedade Brasileira de Zootecnia, 1998a. p.341-343.

FREITAS, A.R.; ALENCAR, M.M.; SILVA, A.M. Ajuste de modelos não lineares em bovinos de corte. II. Influência do mês de nascimento. In: REUNIÃO ANUAL DA SOCIEDADE BRASILEIRA DE ZOOTECNIA, 35., 1998, Botucatu. Anais... Botucatu: Sociedade Brasileira de Zootecnia, 1998b. p.344-346.

FREITAS, A.F.; DURÃES, M.C.; TEIXEIRA, N.M. Curvas de crescimento de novilhas da raça Holandesa mantidas em regime de confinamento. Arquivo Brasileiro de Medicina Veterinária e Zootecnia, v.49, n.1, p.85-93, 1997.

FREITAS, A.F.; DURÃES, M.C.; TEIXEIRA, N.M. Funções de crescimento de animais da raça Holandesa, mantidos em regime de confinamento. In: REUNIÃO ANUAL DA SOCIEDADE BRASILEIRA DE ZOOTECNIA, 32., 1995, Brasília. Anais... Brasília: Sociedade Brasileira de Zootecnia, 1995. p.662-665.

HARTLEY, H.O. The modified Gauss-Newton method for the fitting of nonlinear regression functions by least squares. Thechnometrics, v.3, p.269-280, 1961.

HUGHES, T.E.; PITCHFORD, W.S. Heterosis effects on efficiency of post-weaning growth. In: WORLD CONGRESS ON GENETICS APPLIED TO LIVESTOCK PRODUCTION, 5., Guelph, 1994. Proceedings... Guelph: WCGALP, 1994. v.19, p.197-200.

LAIRD, A.K. Dynamics of relative growth. Growth, v.29, p.249-263, 1965.

LIN, C.Y.; McALLISTER, J.; NG-KWAI-HANG, K.F. et al. Association of milk protein with growth and reproductive performance of dairy heifers. Journal of Dairy Science, v.70, p.29-39, 1987.

MANSOUR, H.; JENSEN, E.L.; JOHNSON, L.P. Analysis of covariance structure of repeated measurements in holstein conformation traits. Journal of Dairy Science, v.74, n.8, p.2757-2766, 1991.

MEZZADRA, C.A.; MIQUEL, M.C. Heterosis and breed transmitted effects in growth curve parameters in Angus Criollo and reciprocal crossbred cows. In: WORLD CONGRESS ON GENETICS APPLIED TO LIVESTOCK PRODUCTION, 5., Guelph, 1994. Proceedings... Guelph: WCGALP, 1994. v.17, p.276-279.

MOODY, D.E.; POMP, D.; NEWMAN, S.; MACNEIL, M.D. Characterization of DNA polymorphisms in three populations of Hereford cattle and their associations with 
growth and maternal EPD in line 1 Herefords. Journal of Animal Science, v.74, p.1784-1793, 1996.

NADARAJAH, K.; MARLOWE, T.J.; NOTTER, D.R. Growth patterns of Angus, Charolais, Chatolais x Angus and Holstein $x$ Angus cows from birth to maturity. Journal of Animal Science, v.59, p.957-966, 1984.

NELDER, J.A. The fitting of a generalization of the logistic curve. Biometrics, v.17, p.89-110, 1961.

OLIVEIRA, H.N. Análise genético-quantitativa da curva de crescimento de fêmeas da raça Guzerá. Ribeirão Preto: Universidade de São Paulo, 1995. 73p. Tese (Doutorado) Universidade de São Paulo, 1995.

OLIVEIRA, H.N.; LÔBO, R.B.; PEREIRA, C.S. Relationships among growth curve parameters, weights and reproductive traits in Guzerá beef cows. In: WORLD CONGRESS ON GENETICS APPLIED TO LIVESTOCK PRODUCTION, 5., 1994, Guelph. Proceedings... Guelph: WCGALP, 1994. v.19, p.189-192.

PEROTTO, D.; CASTANHO, M.J.P.; ROCHA, J.L et al. Descrição das curvas de crescimento de fêmeas bovinas Guzerá, Gir, Holandês x Guzerá e Holandês x Gir. Revista Brasileira de Zootecnia, v.26, p.283-288, 1997.

PEROTTO, D.; CUE, R.I.; LEE, A.J. Comparison of nonlinear functions for describing the growth curve of three genotypes of dairy cattle. Canadian Journal of Animal Science, v.72, p.773-782, 1992.

RATKOSWKY, D.A. Nonlinear regression modeling. New York: Marcel Dekker Inc., 1983. 297p.

RICHARDS, F.J. A flexible growth function for empirical use. Journal of Experimental Botany, v.10, p.290-300, 1959.

ROCHA, J.L.; BAKER, J.F.; WOMACK, J.E. et al., Statistical associations between restriction fragment length polymorphisms and quantitative traits in beef cattle. Journal of Animal Science, v.70, p.3360-3370, 1992.
STATISTICAL ANALYSES SYSTEM - SAS. SAS/STAT 2001: user's guide: statistics version 8.2, CD-ROM. Cary, 2001.

SCHABENBERGER, O. Nonlinear regression with the SAS system. http://www.cas.vt.edu/schabenb/SASNlin.htm. (14 nov. 2001).

SOUZA, G.S. Introdução aos modelos de regressão linear e não linear. Brasília: EMBRAPA-SPI, 1998. 505p.

TAMBASCO, D.D.; PAZ, C.C.P.; TAMBASCO-STUDART, $M$. et al. Candidate genes for growth traits in beef cattle crosses Bos taurus x Bos indicus. Journal of Animal Breeding and Genetics, v.120, n.1, p.51-56, 2003.

TAYLOR, J.F.; COUTINHO, L.L.; HERRING, K.L. et al. Candidate gene analysis of GH1 for effects on growth and carcass composition of cattle. Animal Genetics, v.29, p.194-201, 1998.

UNANIAN, M.M.; BARRETO, C.C.; FREITAS, A.R. et al. Associação do polimorfismo do gene do hormônio do crescimento com a caraterística peso em bovinos da raça Nelore. Revista Brasileira de Zootecnia, v.29, n.5, p.1380-1386, 2000.

Von BERTALANFFY, L. Quantitative laws in metabolism and growth. The Quarterly Review of Biology, v.32, p.217-230, 1957.

WEBSTER, A.J.F. Bioenergetics, bioengineering and growth. Animal Production, v.48, n.1, p.249-269, 1989.

Recebido em: 21/10/02 Aceito em: 16/03/04 